\title{
Paraffinoma of the penis
}

\author{
Stefano Carlo Maria Picozzi • Luca Carmignani
}

Received: 22 March 2010 / Accepted: 5 July 2010 /Published online: 21 August 2010

(C) Springer-Verlag London Ltd 2010

A 38-year-old man, originally from Moldavia, presented at our Emergency Department with edema of the penis, paraphimosis with necrosis of the phimotic ring, glandular ulcer and multinodular hard semimoble penile masses associated with multiple sites of penile and glandular injection (Figs. 1 and 2). The patient reported having undergone penile subcutaneous and glandular paraffin injections $48 \mathrm{~h}$ previously (and also 1 year previously), performed by an untrained

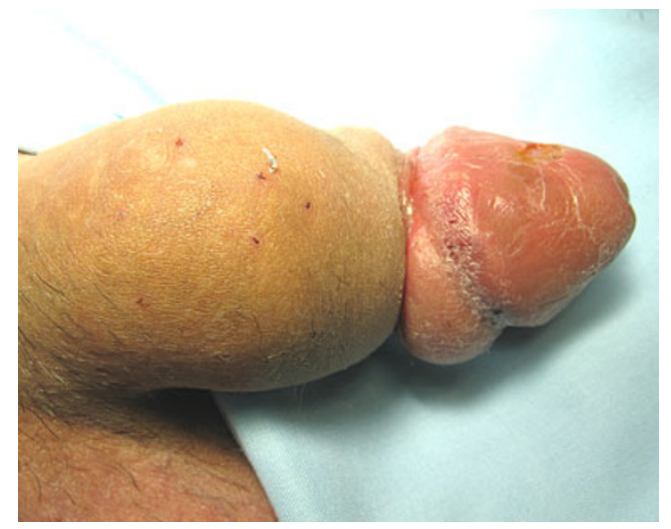

Fig. 1 Multiple sites of penile and glandular injection associated with edema of the penis, para-phimosis and glandular ulcer

Previous reports that might be regarded as redundant publication of the same work: none

The manuscript has been read and approved by all the authors, the requirements for authorship have been met, and each author believes that the manuscript represents honest work.

S. C. M. Picozzi $(\triangle) \cdot$ L. Carmignani

Urology Department, IRCCS Policlinico San Donato,

University of Milan,

Via Morandi 30,

20097 San Donato Milanese, MI, Italy

e-mail: stepico@tin.it

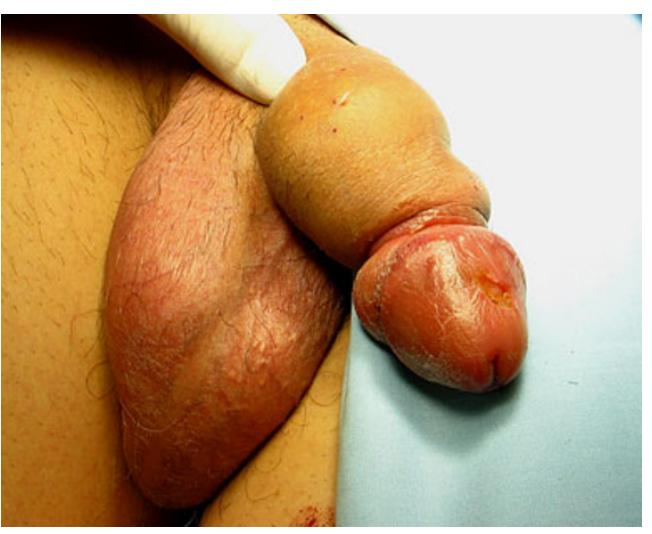

Fig. 2 Multiple sites of penile and glandular injection associated with edema of the penis, para-phimosis and glandular ulcer

person, for augmentation of the penile contour. Magnetic resonance imaging showed that mineral oil was distributed outside of and attached to Buck's fascia, the corpus cavernosum, corpus spongiosum and in the glans (Figs. 3 and 4). Urgent circumcision with evacuation of the oil and partial resection of the paraffinoma was performed. Postoperative recovery was uneventful, and the patient was discharged on the 2nd day after surgery.

The first report of paraffin injection into the male genitalia was by Gersuny in 1899; in this case mineral oil was injected into the scrotum of a boy who had undergone bilateral orchiectomy for genital tuberculosis [1]. The body lacks the enzymes to metabolize interstitial exogenous oils, and a foreign-body reaction occurs, so paraffinomas consist of a granulomatous foreign-body reaction inducing a sclerosing lipogranuloma. Adverse events from the injection of these oily substances are well known and were reported as early as 1906 in two patients who had received paraffin injections for facial wrinkles and developed disfiguring subcutaneous nodules [2]. Despite the severe destructive consequences, 


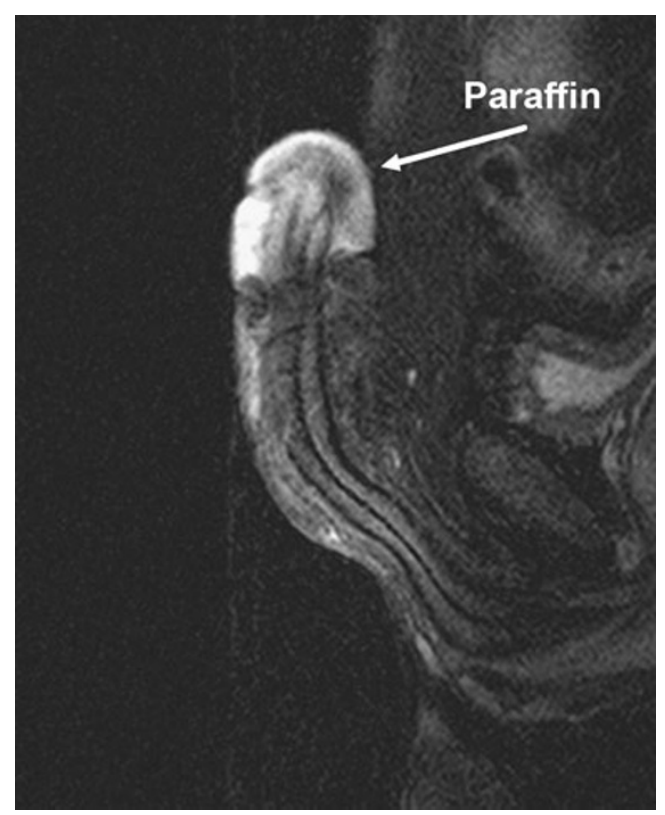

Fig. 3 Magnetic resonance imaging showing the mineral oil distribution

this practice continues in some parts of the world, such as Asian and Eastern European countries.

Although paraffinomas are certainly not common, clinicians need to be aware of this entity, especially because of the increasing number of migratory fluxes. Most complications require urologists because of the possible immediate danger to the penile structures.

\section{Acknowledgement None}

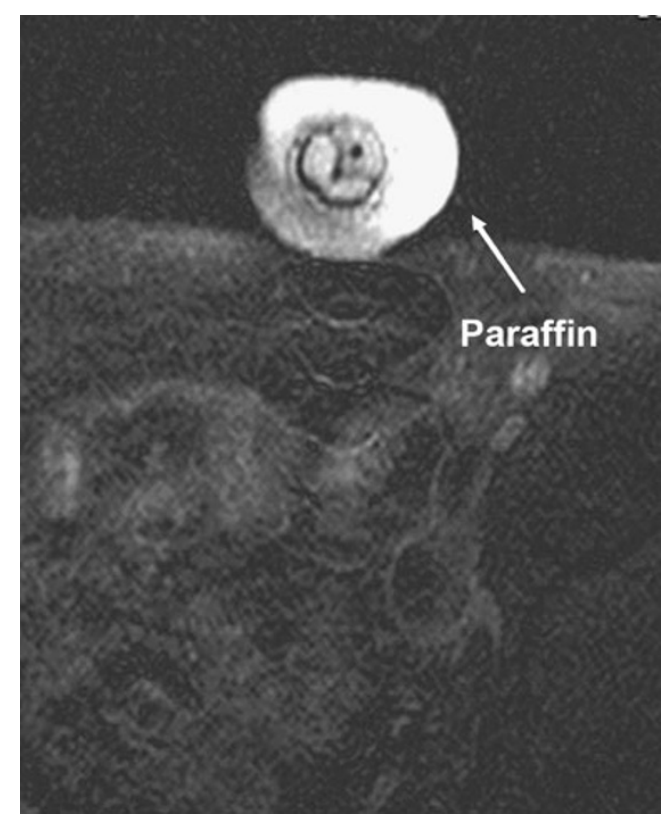

Fig. 4 Magnetic resonance imaging showing the mineral oil distribution

\section{Conflicts of interest None}

\section{References}

1. Gersuny R (1903) Harte und weiche Paraffin prosthesen. Zentralbl Chir 30:1

2. Heidingsfeld ML (1906) Histopathology of paraffin prosthesis. J Cutan Dis 24:513-21 